\title{
Policy-based Self-healing for Radio Access Networks
}

\author{
Javier Baliosian ${ }^{1,2}$, Katarina Matusikova ${ }^{2}$, Karl Quinn ${ }^{2}$ and Rolf Stadler ${ }^{3}$ \\ ${ }^{1}$ School of Engineering, University of the Republic, Montevideo, Uruguay. \\ ${ }^{2}$ Ericsson Ireland Research Centre, Athlone, Ireland. \\ ${ }^{3}$ Laboratory for Communication Networks, KTH Royal Institute of Technology, Stockholm, Sweden. \\ Email: javierba@fing.edu.uy, \{katarina.matusikova, karl.quinn\}@ericsson.com, stadler@kth.se
}

\begin{abstract}
Various centralized, distributed or cooperative management systems have been proposed to address the demands of wireless telecommunication networks. However, considering the size, complexity and heterogeneity that those networks will have in the future, current solutions either do not scale properly, or have no support for automation, or lack of the flexibility and simple control that operators will need for managing future networks in a costeffective way. To address this problem, we designed Omega, a distributed and policy-based network management system that uses rich knowledge-modeling techniques to develop self-configuration capabilities. Omega also implements a novel conflict-resolution method that uses high-level goals and machine learning techniques to optimize its policy-based decisions. Using simulations, in this paper we show how Omega reduces the impact of a node crash on the overall availability of a radio access network by optimizing the lists of neighboring cells of the nodes in the vicinity.
\end{abstract}

\section{INTRODUCTION}

The current proliferation of radio access technologies is leading to the emergence of heterogeneous, complex and dynamic communication systems. In this context, the centralized paradigms widely adopted to handle FCAPS functionalities (Fault, Configuration, Accounting, Performance and Security) are starting to show their inherent weaknesses in scalability, flexibility and robustness. However, most of the existing attempts at network management decentralization are rigid and hierarchical solutions that cannot modify their structure dynamically or reallocate management tasks in order to adapt their configuration to network changes. In addition, decentralized approaches rely mostly on simple agents without operational, configuration state or context knowledge and, therefore, with very limited decision-making abilities. All those limitations remain a major obstacle for a costeffective exploitation of networks by telecom operators tackling, in a timely fashion, predictable or unpredictable failures.

This paper first presents an overview of an ontology-based implementation of Omega [1] a distributed, self-management architecture that addresses those limitations (§ II-A-II-B), and second, shows how the system optimizes and heals autonomously some configuration aspects of a UMTS Radio Access Network (RAN) ( $(\mathrm{IV})$. This optimization process is carried out in a distributed manner using a novel goal-oriented policy conflictresolution process. This process tries to predict the consequences of choosing one policy from among several conflicting policies maximizing the probability of reaching the strategic goal of the system (§ III).

\section{SySTEM ARCHITECTURE}

\section{A. Network Architecture}

Omega combines three common organizational structures centralized, hierarchical and fully distributed- to address differ-

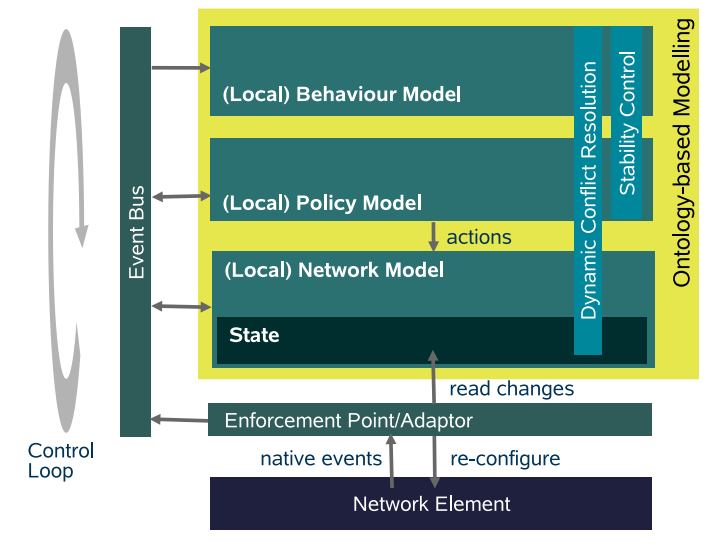

Fig. 1. Node Architecture

ent tasks with the most fitting approach while trying to maximize the distribution of tasks over the nodes. Only the tasks that inherently require a centralized organization, such as accounting, or those tasks which perform better given a weakly distributed structure, are carried out using hierarchical structures. Other management tasks such as local optimizations or on-demand discovery of resources follow a fully distributed approach.

\section{B. Node Architecture}

The Omega node architecture (see Fig. 1) enables autonomy with a novel combination of technologies. First, task automation and current node status are coupled in a single ontology model, a semantically enriched Management Information Base (MIB) (Section II-C). Hence, the MIB not only tracks current configuration but provides the workflows to change that configuration. Second, the decision-making capability (Section II-D) is provided by a policy engine which allows high-level decisionmaking based on operators' preferences and strategic decisions. The finer granularity checks and balances of the rule engine are provided by the ontology model MIB which specifies the constraints on possible configuration actions imposed by the nature and current status of a node. Released from this low-level checking functionality, the policy engine can impose strategic operator-driven choices on configuration activity. Finally, each Omega node monitors the effects of its own configuration actions over long timescales (Section II-E) adapting policy decisions on the basis of observed effects (Section III).

\section{Automating actions: Network Model}

The Network Model is an ontology-based enhanced Management Information Base (MIB). The local Network ontology model stores the current configuration of the local device and knowledge of its relevant network state. This includes its rela- 


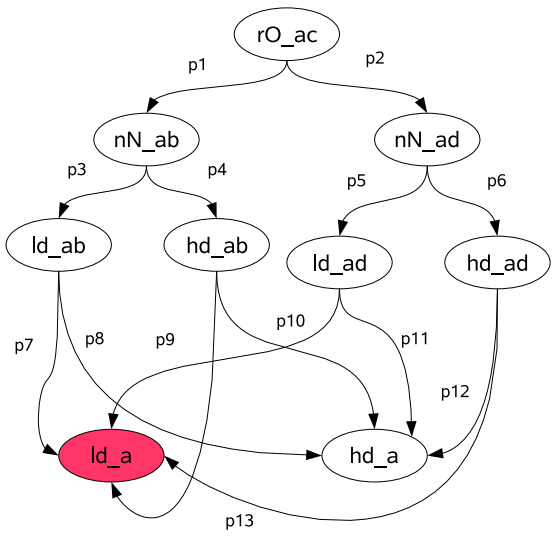

Fig. 2. Behavior Model based on Bayesian Networks (nN represents event newNeighbor, rO remOverlap, hd highDrops and ld lowDrops) tionships with other objects in the network and configuration constraints. It also stores the workflows associated with configuration tasks and has the ability to executing them on the local or remote MIBs. The Network Model therefore is both an information repository and an active propagator of change to this repository. Each node in an Administrative Domain has its own local Network Model instance storing its own current configuration, constraints and relationships. The sum of these local models is a Global Network Model. Since the local Network Model instances are not necessarily consistent with each other, the Global Network Model instance may be internally inconsistent. The trade-off between perfect consistency and traffic overhead is governed by consistency mechanisms that are outside the scope of this paper.

\section{Taking decisions: Policy Decision Point}

Within this framework, policies are implemented as conditionaction rules where the condition may be the occurrence of some the events (e.g., an alarm or a service request), in a certain network Resolution component of an Omega node is designed to adapt state and the action is the desired response to that condition those TFs to current network conditions by incorporating the event, as defined by the operator. The Policy Model includes a probabilities of event occurrences derived from the Behavior Policy Decision Point (PDP), which subscribes to events through Model component. When the PDP evaluates a TF for a specific an event notification service (not described in this paper) and policy condition it, does so using the network state encoded in the evaluates the policy conditions using the information stored in Network Model (NM). Once the set of possible actions to trigger the Network Model. The reconfiguration actions decided by is identified (because their respective conditions have positive the PDP may also involve other network elements, in which TFs) the Dynamic Conflict Resolution component computes case the node may be the manager of the activity and delegate the probability for the system's goal encoded in the Bayesian responsibility for some actions to other nodes. The policies are network and uses that value as the TF for the actions. In this way, modeled using a special kind of finite state machine with input each conflicting policy is associated with a final numerical value and output, presented in [2], called Finite State Transducers based on the condition TF computed using the network state extended with Tautness Functions and Identities (TFFST). This represented by the NM, and a predicted estimate of policy effects model is oriented to the resolution of policy conflicts as each expressed by the action TF computed using the knowledge in condition and action has an associated Tautness Function (TF), a the BM. This combination of operator and system experience sort of cost-function that gives dynamic priorities to conflicting combination have not been seen in the literature and seems a policies depending on the data stored in the NM.

\section{E. Learning from experience: Behavior Model}

In a distributed self-managed network, devices perform independent actions in order to fulfill services and meet user requirements. This independent behavior may affect the network as a whole. Therefore, in order to detect and possibly predict behavior that will adversely affect the provision of services and the experience of end users, it is critical to observe the behavior of events and messages within the network as a whole.
Performance and Fault Management are standard systems in network status. The ability to infer the impact of how a network operates from what the network is doing is very useful to a extrapolate what may happen in the network based on knowledge about what has happened in the network in the past. The Omega Behavior Model is designed to carry out these monitoring, of the Omega Behavior Model is a Dynamic Bayesian Network [3] implemented using the Web Ontology Language (OWL). Bayesian Networks consist of a Directed Acyclic Graph (DAG) domain, in this case events in a network (parameters, alarms, configuration requests), and the arcs represent the influential Bayesian network, we are especially interested in those associ-

\section{Adapting to Change: Dynamic Conflict RESOLUTION}

At run-time, conflicts may occur between policies which could not be detected at compilation time, as they arise from the has evaluated the conditions of its local policies and two or more policies could be triggered by the same recent events. most appropriate to current circumstances. In a classic PDP, the engine applies a system of priorities associated with each policy
in order to select between them. The TFs mentioned in $\S$ II$\mathrm{D}$, are a more dynamic way of prioritizing policies, but they are still coded before policies are deployed and may not encode promissing approach, as far as the machine learning techniques used are light and scale properly in devices that have real-time networking tasks as main purpose.

Then the conflict can be resolved as usual using the priorities given by the TFs. This process maximizes the probability of reaching the system goal given policies of varying specificity.

\section{Neighboring Cells List SElF-HeAling}

Each cell in a UMTS network has a list of neighboring cells, which are specifically some of the cells in the vicinity 

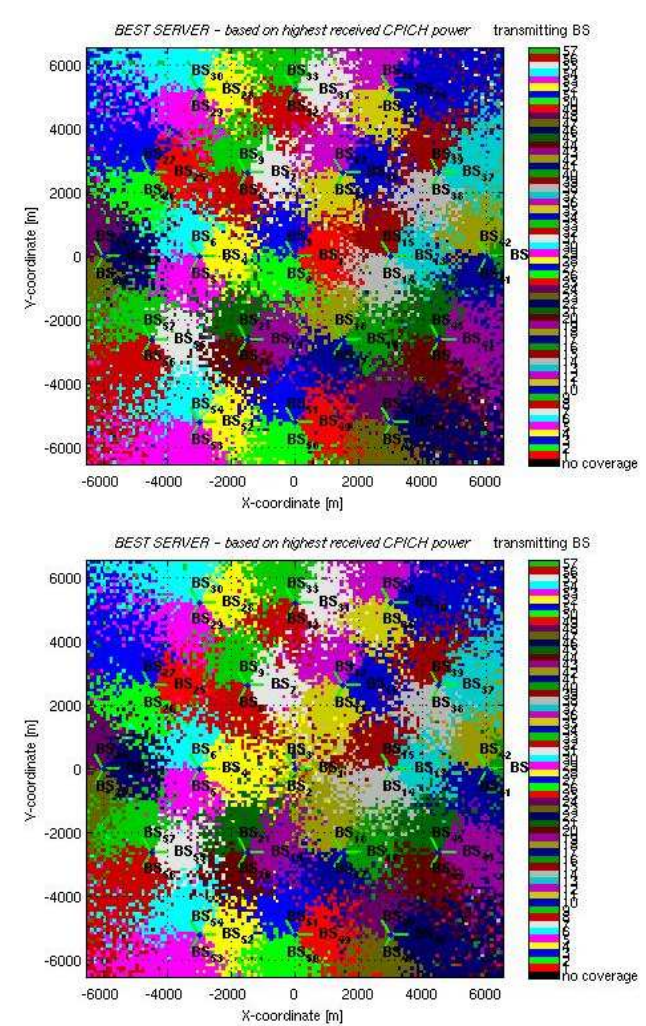

Fig. 3. Cell Coverage Areas Before and After the RBS Crash.

with overlapping coverage regions to which it is possible to perform an intra- or inter-system handover processes. Today, this neighboring cells list is an output of the network planning process. These lists are then configured statically in the Radio Network Controllers (RNC), which are entities that control large sets of Radio Base Stations (RBS), the wireless access points. RNCs and RBSs are entities of the Universal Terrestrial Radio Access Network (UTRAN) [4].

The case in which a cell or an entire RBS disappears is a problem specifically in some regions with large and unreliable power-distribution or telecommunication infrastructure. Therefore, the ability to rapidly and automatically recover from such failures -at least temporarily and to a certain extent- is crucial. We address that need as an exercise for the Omega soution following the framework presented in [5]. This particular application is a self-healing procedure in which the RBSs near a failed base station, in a coordinated and autonomous fashion, reconfigure their respective neighboring cell list to reflect the new network topology supporting efficient handover and avoiding call drops. An up-to-date view of the network topology at each node is maintained using DOC, the protocol for the discovery of overlapping cells presented also in [5]. We carried out the experiments in a simulated environment that is described below.

\section{A. Simulation Environment}

In order to achieve a realistic simulation environment, we to the terminals that where connected to the disappearing cells. choose three separate simulation packages, which in combination Additionally is possible to see in that graph how the number and through interaction achieve an environment that allows us of handovers drops significantly. This is caused by a list of to take into account the dynamic aspects of radio coverage, neighboring cells that no longer reflects the network reality. After terminal mobility and the execution of our distributed protocol. the crash happens, DOC protocol updates the overlapping cell We have implemented DOC and the required network entities information at the surrounding RBSs (as seen in the Fig. 4(b)) on JavaSimulation [6] , a DES simulation package. The radio triggering remOverlap events (depicted as rO in Fig. 2) which, 
in turn, triggers again the same policy-driven neighboring cell configuration process. The corresponding newNeighbor events are triggered and the BM is again fed with this information. The view of the network for the cell 18 is depicted in the Fig. 4(b). In that figure it is possible to appreciate how, after the RBS crash, two cells that were previously overlapping with cell 18 but not configured as neighbors (not in green) are now re-configured as neighbors of cell 18 .

\section{Policy Model}

The policy model used to perform the neighboring cells list configuration mentioned in the scenario is quite simple. The following are the constraints, rules and goals of the system deployed at each node.

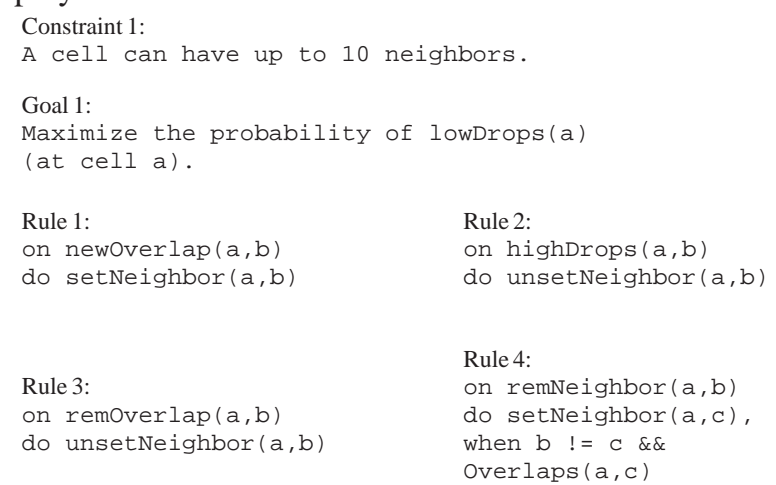

The events newOverlap $(a, b)$ and removerlap $(a, b)$ are issued by DOC meaning that was discovered an overlap between cells $a$ and $b$ and that an existent overlap between cells $\mathrm{a}$ and $\mathrm{b}$ has disappeared. The higher-level event remNeighbor $(a, b)$ is issued when the neighboring relationship between cells $\mathrm{a}$ and $\mathrm{b}$ has been erased. The notifications setNeighbor $(a, b)$ and unsetNeighbor $(a, b)$ are issued by the PDP asking to the NM the configuration of $a$ and $b$ as neighboring cells. This task implies a transactional configuration process of both nodes that is out-of the scope of this paper. The event highDrops $(a, b)$ is issued by the Enforcement Point/Adapter component (see Fig. 1) when the number of call drops on handovers between cells $a$ and $b$, normalized to the number of handovers between those cells and to the total number of handovers of the local cell, exceed a given threshold.

In the following we describe how this set of policies, constraints and goals together with the conflict resolution process described before build up a process that chooses as neighbors those cells that minimize the probability of having call drops.

1) Optimization Process: The optimization process with which the best overlapping cells in the vicinity are added to the list of neighboring cells is a trial and error procedure. After the system is initialized newOverlap events are issued and Rule 1 is executed trying to configure each of the new overlapping cells also as neighboring cells until the number of configured neighbors reaches the limit imposed by Constraint 1. Eventually, one of the cells chosen as neighbor may issue a highDrops event. This executes Rule 2 and the cell is erased of the list of neighboring cells, a procedure that causes a remNeighbor event. This last event causes the execution of Rule 4, which tries to add all overlapping cells that are not the erased one to the list of neighboring cells. This causes a dynamic conflict with

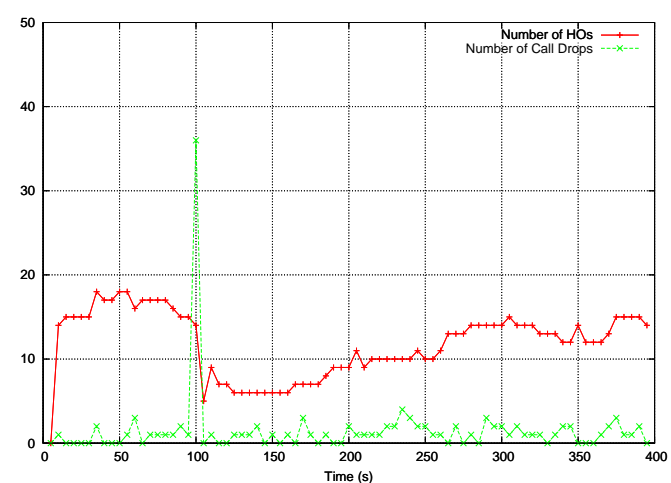

Fig. 5. Handovers and call Drops at Cell 18

Constraint 1 when all those new neighbors, plus those already configured, are more than 10 . The goal-oriented conflict resolution process will then choose as new neighboring cells those overlapping cells that maximize the probability of a lowDrops notification following the probabilities computed by the Behavior Model.

2) Self-healing Process: In front of a cell faillure, the Omega nodes react in a similar fashion to the optimization process. When a cell disappears, DOC issues a remOverlap notification. This event triggers Rule 3 which unsets the cell as neighbor at each of the nodes in the vicinity. Consequently, remNeighbour events are issued and the distributed optimization process described before starts again trying to re-optimize the list of overlapping cells of each affected node. In Fig. 5 it is possible to see how this combination of rules and continuous conflict resolutions causes that after the RBS crash, the number of handovers recovers progressively to a value near the one before the crash.

\section{COnClusion}

In this paper we briefly describe Omega, a novel architecture for self-management of Radio Access Networks. We also present an overview of its novel goal-oriented conflict resolution methodology. And finally, we show how this system addresses a problem related to the reliability of current UMTS networks. With the preliminary work presented, we have shown how it is possible to perform a self-healing policy-based process that uses the system's own experience to temporarily alleviate the effects of an RBS faillure.

\section{REFERENCES}

[1] J. Baliosian, H. Oliver, A. Devitt, F. Sailhan, E. Salamanca, B. Danev, and G. Parr, "Self-configuration for radio access networks," in IEEE Policy 2006, 5-7 June 2006, p. 4pp.

[2] J. Baliosian and J. Serrat, " Finite State Transducers for Policy Evaluation and Conflict Resolution ," in Proceedings of the Fifth IEEE International Workshop on Policies for Distributed Systems and Networks (POLICY 2004), June 2004, pp. 250-259.

3] A. E. Nicholson, "Monitoring discrete environments using dynamic belief networks," Ph.D. dissertation, Department of Engineering, Oxford, 1992.

[4] 3GPP, "TS 25.401, UTRAN overall description," 2007. [Online]. Available: http://www.3gpp.org/ftp/Specs/html-info/25401.htm

5] J. Baliosian and R. Stadler, "Decentralised configuration of neighbouring cells for radio access networks," in Proceedings of the IEEE International Symposium on a World of Wireless Mobile and Multimedia Networks, WoWMoM 2007., Helsinki, Finland, June 2007.

[6] K. Helsgaun, "Discrete event simulation in java," Roskilde University, Tech. Rep., 2000.

[7] J. Laiho, Radio Network Planning and Optimisation for Umts, A. Wacker and T. Novosad, Eds. New York, NY, USA: John Wiley \& Sons, Inc., 2002. 